\title{
Phase transformation in white etching area in rolling contact fatigue
}

\author{
Yun-Shuai Su, Shu-Xin Li ${ }^{*}$, Si-Yuan Lu, and Li-Biao Wan \\ School of Mechanical Engineerying and Mechanics, Ningbo University, Ningbo 315211, PR China
}

\begin{abstract}
Rolling contact fatigue (RCF) involves microstructural change in the subsurface of contact. The changed microstructure is generally termed as white etching area (WEA) as it appears white under optical microscope when etching in nital solution. WEA has been acknowledged as one of the primary failure modes in RCF since it causes severe local inhomogeneity of microstructure. It was reported that WEA consists of nano ferrites as martensite grains and carbides are significantly refined in the WEA. Some carbides are dissolved. In some cases, an amorphous-like structure was occasionally observed in the WEA, indicating that phase transformation may possibly occur. The WEAs were studied by using scanning electron microscopy (SEM), transmission electron microscopy (TEM) and Electron back scattered diffraction (EBSD). The result showed that WEA is dominated with an amorphous phase with martensite, austenite and carbides embedded interior. A distinct interface between the matrix and the WEA was present. In addition to grain refinement down to nanometers, phase transformation including amorphization and austenitization happened in WEAs. The content of austenite was increased from $2 \%$ in the matrix to $20 \%$ in the WEA. The analysis showed that phase transformation is controlled by plastic deformation mechanism.
\end{abstract}

\section{Introduction}

The rolling bearings are always failed under rolling contact fatigue with much shorter lives than that designed, especially in wind turbine gearbox bearings. Plastic deformation is highly localized into a narrow region at both contact surface and subsurface until the number of cycles reaches a certain level. When inspecting cross-sections of failed bearings or samples after RCF using optical microscope, white etching matters such as white etching bands [1,2], white etching areas (WEAs) [3-7] and white etching layers (WELs) [8] are observed in different depths below the contact surface. These white microstructural changes are different from base materials in terms of compositions and hardness.

White bands and WEAs are commonly observed in bearing steels and have been extensively studied, especially WEAs [1-7]. As a result of microstructural alteration, WEA has been accepted as one of the primary flaking failure mode in RCF since it causes severe local inhomogeneity of microstructure and cracks associated. Extensive investigations have been carried out on WEAs regarding microstructural evolution and formation mechanisms. The results showed that WEAs are composed of nano-sized ferrites $[1,2,5]$. The hardness of WEAs varies with materials. They are harder than the base materials of martensitic steels [9-11], but softer than the base materials of carbide-free nano-sized bainitic steels [12]. RCF is a cyclic dynamic loading mode involves repeated rolling and sliding. Shear morphology present in WEA such as elongated carbides [5] once plastic deformation accumulates to an extent level during RCF. WEA composed of nano-sized ferrites with grain size of tens nanometers. Some authors proposed that WEAs may possible form as a result of grain refinement under mechanism of dynamic recovery or low temperature recrystallization [5,9]. An amorphous-like region was also presented in WEA except the nanocrystallites $[9,10]$. We have provided the evidence for an amorphous phase exist in the WEA $[3,4]$.

Although WEAs have been studied extensively, but an in-depth comparison of this deformed matter has rarely been given. This paper presents a better understanding of the composition in the WEA and analyse the formation mechanism in detail. There may have relations since they are both the result of microstructural changes in bearing steel under RCF.

\section{Materials and methods}

Corresponding author: 1 i shuxin@ $163 . c o m$ 
The failed sample with WEAs in this paper is tempered martensite steel of AISI52100 with the main chemical composition of (wt.\%): C, 0.09; Cr, 1.87; Si, 0.31; Mn, $0.32 ; \mathrm{P}, \leq 0.027 ; \mathrm{S}, \leq 0.020 ; \mathrm{Fe}$, balance [3]. The materials were austenitized at $860^{\circ} \mathrm{C}$ for 2 hours and quenched in oil to room temperature, subsequently followed by tempering at $160{ }^{\circ} \mathrm{C}$ for $1 \mathrm{~h}$. This material consists of matensite, bainite and retained austenite (less than 2 vol\%). The tests were carried out on a RCF machine with various SRRs of 5\%-20\% and contact pressure ranging from $0.9 \mathrm{GPa}$ to $2.0 \mathrm{GPa}$.

The RCF sample bearing were sectioned along circumferential and axial direction. The inspection was conducted using scanning electron microscopy (SEM), transmission electron microscopy (TEM), and electron backscattered diffraction (EBSD) and hardness test. Especially, focused ion beam (FIB) installed in SEM was used to prepare a sample for TEM analysis from the deformed location.

\section{Results}

\subsection{SEM morphology of WEA}

Fig. 1(a) displays a typical WEA at the depth of $810 \mu \mathrm{m}$ below the contact surface [4]. It has inclined to 45 degrees with the contact surface. According to the researches of others, WEA can be formed at any angle with the contact surface [13-16]. WEA was distributed along the crack and split into two parts with the widest part of $2.6 \mu \mathrm{m}$ on the left side. The microstructure next to the WEA does not show an obvious difference with the matrix except slight sheared morphology. Fig. 1(b) shows another WEA initiated at non-metallic inclusion, some researchers call it butterfly [7,9]. The cracks initiated around inclusion and propagated parallel to contact surface. Besides, there is another WEA distribution along the cracks which propagated parallel to roller surface, as shown in Fig. 1(c).

\subsection{Stress-Number of cycles curve}

Fig. 2 presents the maximum contact pressure versus the number of cycles of RCF. It can be seen that the RCF lives decrease as the maximum contact pressure decreases with the exception of the point at $1.5 \mathrm{GPa}$ with the SRR of $5 \%$. WEA was observed at various contact pressure levels as marked in Fig.2. The effect of the SRR on the formation of WEA exhibits much scatter. WEA was formed at the contact pressure of $0.8 \mathrm{GPa}$ with the SRR of $10 \%$, but was absent at $1.0 \mathrm{GPa}$ and $15 \%$. The same phenomenon occurred at $1.2 \mathrm{GPa}$ and $10 \%$ and 1.4 $\mathrm{GPa}$ and $15 \%$, where WEA was observed under the former condition but not under the latter. This indicates that a higher contact pressure and a larger SRR do not necessarily generate WEA as the formation of WEA depends not only on stress and deformation state but on local material microstructure as well. Coincidently, WEA was observed at all SRRs of $10 \%$ regardless of the contact pressure level.
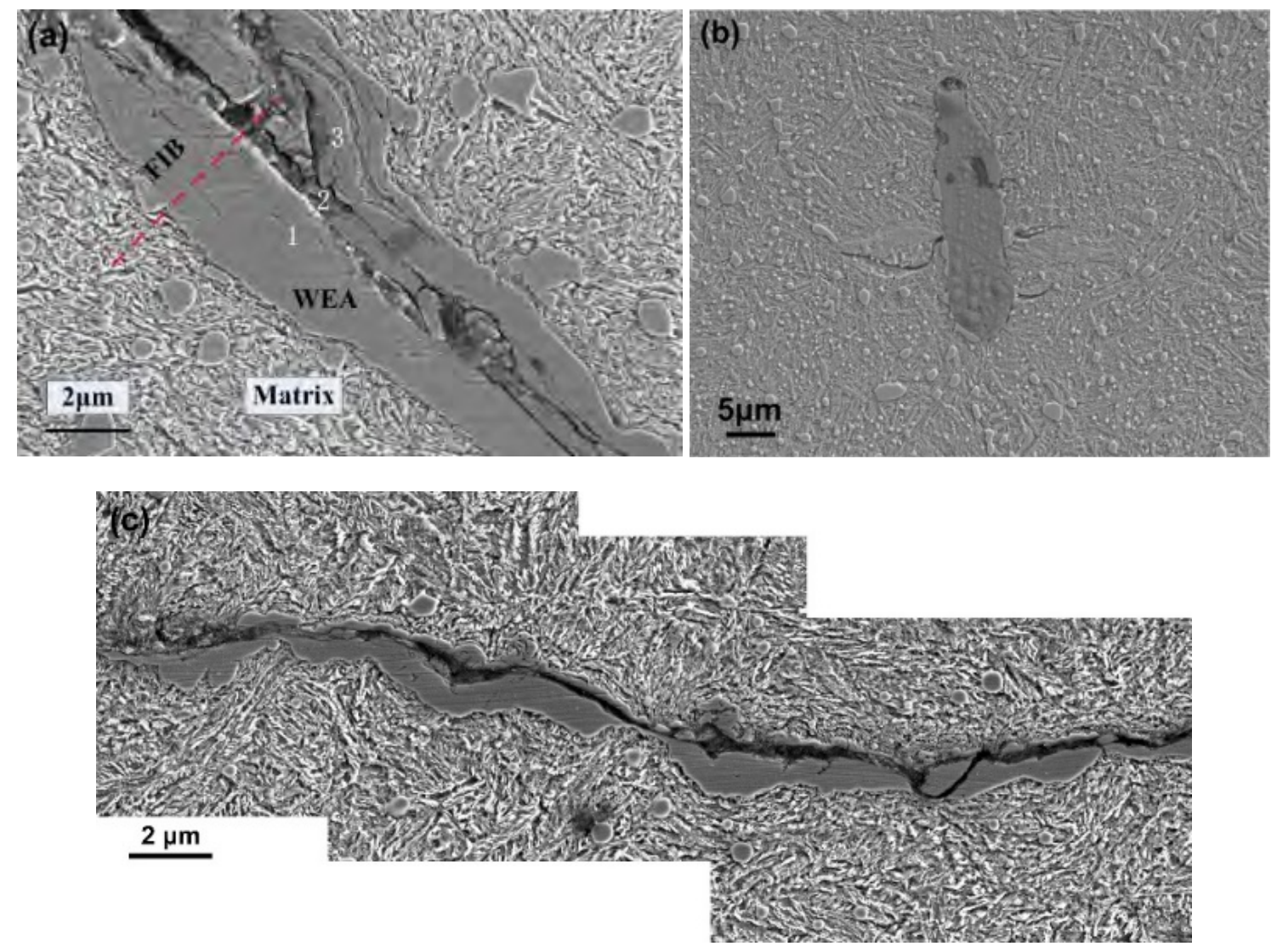
Fig. 1. SEM images (a)WEA along the crack [4]; (b) WEA initiated at non-metallic inclusion; (c) WEA propagate along the crack which parallel to contact surface

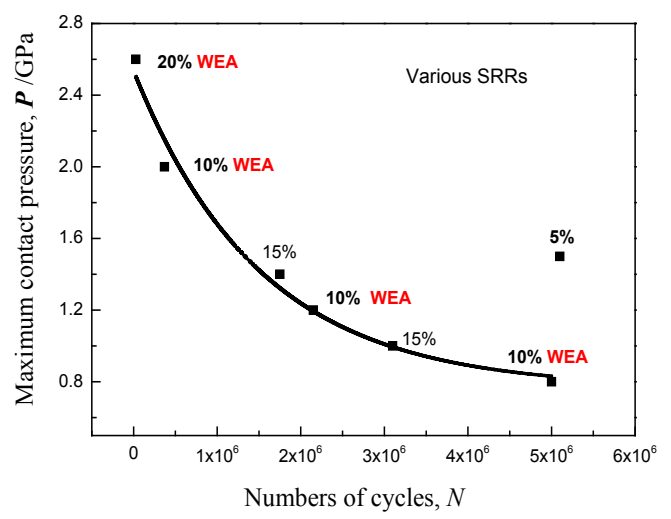

Fig. 2. Contact pressure versus the number of cycles of RCF at various SRR

\subsection{TEM microstructure}

TEM sample of WEA were taken from designated location as showed in Fig. 1(a) and the bright field TEM images in Fig. 3. The deformed matrix consists of refined grains and deformed matrix, such as Fig. 3(a). Grains were refined compared to the base material. We can hardly identify the original tempered martensite and the microstructure filled with dislocation cluster as circled. The size of the grains in WEA refined down to nano-sized ranging from $3 \mathrm{~nm}$ to $50 \mathrm{~nm}$, as shown in Fig. 3(b). The WEA and matrix have different microstructure and the carbides were disappeared. The sheared morphology is no longer exist in the WEA and it contains amorphous phase based on deep inspection, such as Fig. 3(b).

Fig. 3(a) also shows the selected area diffraction (SAD) patterns in order to further understand the composition of the WEA. The thick halo ring that occurred in area "B" indicated an amorphous structure, which is different from "A". Fig. 3(b) is the HRTEM image of amorphous structure found in the WEA around place "B" in Fig. 3(a). Besides, martensite $\alpha$ ', austenite $\gamma$, and carbide $\theta$ were indexed in the WEA [4], which is also different with the SAD pattern in the matrix.

\subsection{EBSD inspection}

The size and the shape of grains can be measured by EBSD. Besides, phase identification and percentage calculation can also be performed. Fig. 4 showing the EBSD-orientation maps of the WEA and matrix, grains size distribution was also presented. The grains were seriously refined in the WEA compared with the matrix, as shown in Fig. 4(a). The WEA present an amorphous feature. The sizes of the matrix near the WEA were found from approximately $100 \mathrm{~nm}$ to $600 \mathrm{~nm}$ and the WEA filled with the size of $30 \mathrm{~nm}$ particles, as described in Ref [4]. Fig. 5 gives the EBSD phase maps in WEA. The red is austenite and the green is martensite. The content of austenite in WEA is $20 \%$ and in the matrix is $2 \%-3 \%$. It shows that the content of austenite in the WEA is much higher than matrix. Austenite is uniformly distributed in WEA.
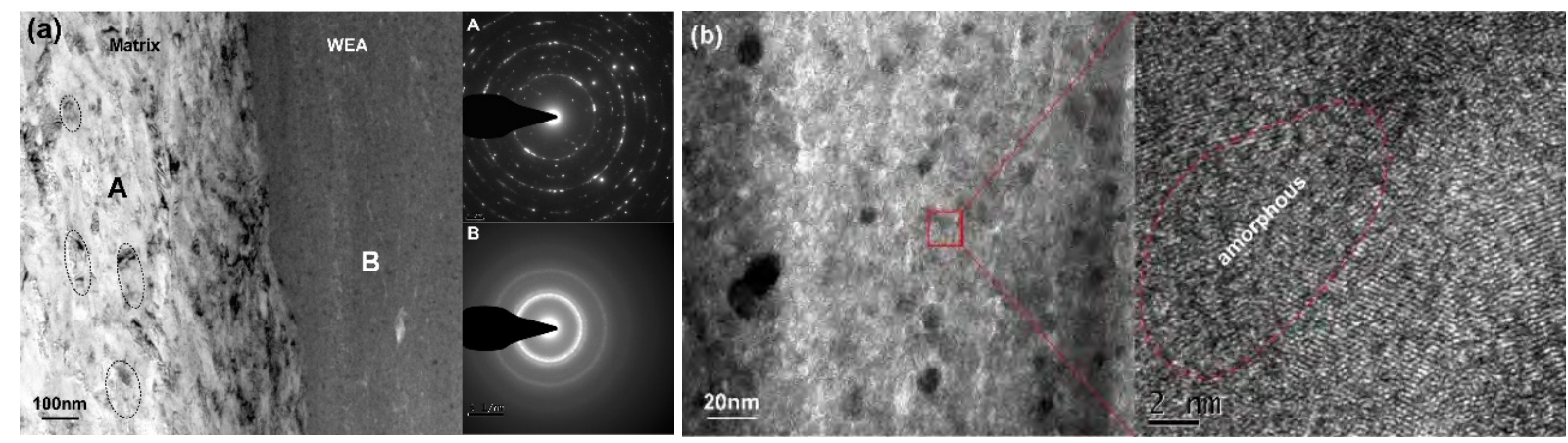

Fig. 3. TEM images (a) Interface between the matrix and the WEA; (b) HRTEM image of morphous structure in the WEA 

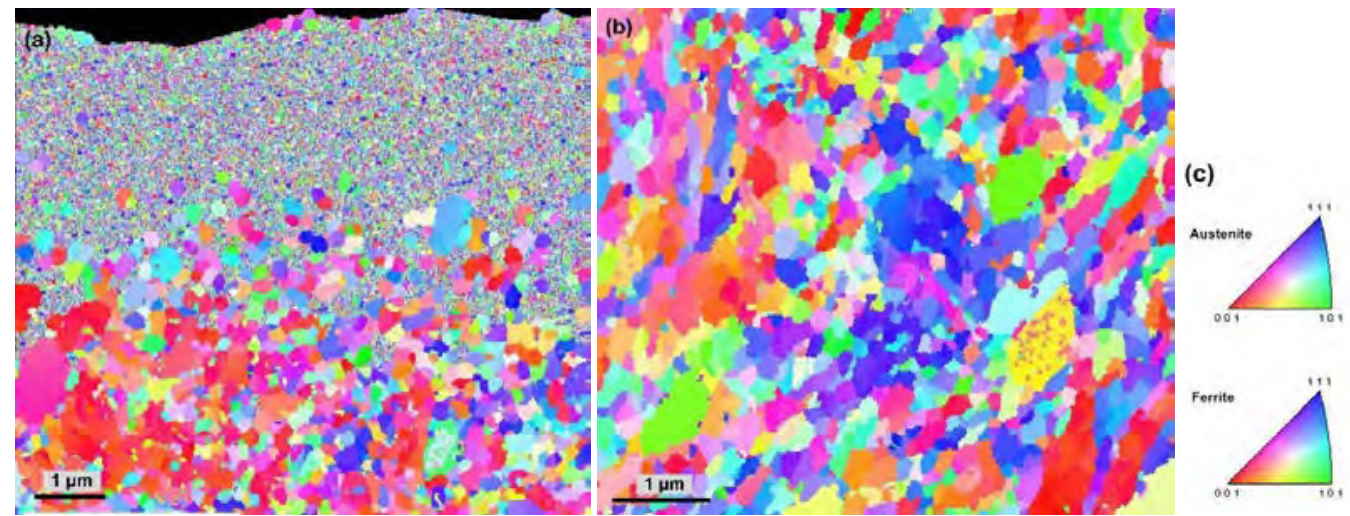

Fig. 4. EBSD-orientation maps [4] of (a) the interface; (b) the matrix; (c) standard inverse pole figure maps

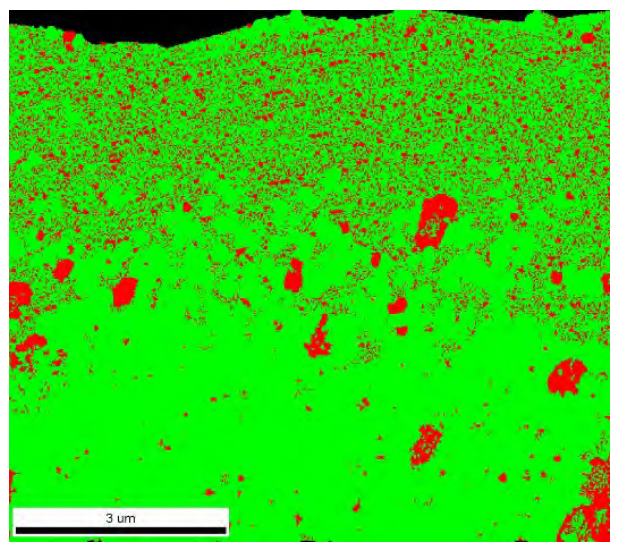

Fig. 5. EBSD phase maps with martensite bcc colored green and austenite fcc colored red

\section{Discussion}

\subsection{Contact pressure threshold for WEA formation}

The P-N diagram (Contact Pressure-Number of cycles) proposed by Warhadpande et al [12] suggested that there exists a threshold contact pressure of $2.5 \mathrm{GPa}$, below which no microstructural change would occur in RCF. WEA was largely observed under the contact pressure of 2.5-5.6 GPa $[17,18]$. However, in some cases where WEA is formed at a low contact pressure of 1-2 GPa, sliding has to be introduced. A typical example was given by Evan's et al [7] that WEA was generated at 1.2 GPa with the SRR $14.8 \%$. A large number of WEAs were found at $1.9 \mathrm{GPa}$ with the slip ratio of $-30 \%$ [13]. But no WEA was formed in the positive sliding even when the SRR reached $30 \%$ and only cracks were produced, implying that microstructure alternation does not happen in positive sliding. However, this observation conflicts with Evan's [7] and our current result that WEA forms in the positive sliding direction. The current result shows that WEA can be generated at the contact stress of $0.8 \mathrm{GPa}$ with the SRR of $10 \%$. In view of the above discussion, it is rational to suggest that it is the combined co-action of the contact pressure and the sliding that determines the formation of WEA. The threshold of the contact pressure may not exist under the current RCF condition. As mentioned above, WEA is the result of microstructural alternation. Its generation is significantly dependent on local material microstructure. This can be proved that a higher contact pressure with a larger SRR (for example, comparing the points of $1.2 \mathrm{GPa}$ and $10 \%$ (WEA) and $1.4 \mathrm{GPa}$ and $15 \%$ (without WEA) in Fig.1) did not generate WEA.

\subsection{Grain refinement in the WEA}

Grains in the WEA were refined to nano-sized and grains next to the WEA were refined to micro or nano scaled based on TEM inspection. It is reported that the materials would became unstable under certain loading condition in Ref. [19]. Under the large deformation, grains are refined through disintegrating and breaking down the elongated grains by entangled dislocation clusters. The process is also accompanied with splitting and twisting, which can further accelerate the refinement, as indicated in Fig. 3(a). Dougherty et al [20] stated that shear localization is a mode of stress accommodation, plastic flow is confined to zones of intense shear surrounded by regions of lower, more homogeneous plastic deformation.

Multiple mechanisms were proposed to explain this phenomenon, one of which stated that the white etching 
matter formed around a subsurface crack is a symptom of crack faces rubbing which leads to local changes in the material microstructure [21]. There was a strong evidence that deformed microstructure dominate the WEA, supporting the theory that it is a deformationinduced but not a thermal-induced process.

\subsection{Phase transformation and amorphization in the WEA}

The amount of austenite increased a lot and consists of an amorphous phase indicated that phase transformation occurred in the WEA. This essential microstructural change appeared during the formation of the WEA compared to previous study, which consist of nanocrystalline ferrites $[5,9,22]$. It is generally accepted that the existence of austenite provided an evidence of phase transformation $[23,24]$.

Baumann [25] stated that temperature can induce ferrite transformed to austenite. Pyzalla [26] reported that the transformation from ferrite and pearlite to austenite occurred due to austenitization, and austenite transformed to martensite during cooling. The heat that raise up the temperature comes from the friction during the sliding of two contact surfaces. The WEA were not generated at the surface even though the highest temperature appeared at top surface [4]. And the temperature cannot reach the austenitization temperature of $720{ }^{\circ} \mathrm{C}$ because lubrication play a role in cooling during RCF. Another phenomenon that WEA formed with various depth below the surface support that the friction of contact surface cannot became the formation condition of WEAs.

Phase transformation has been proved under mechanically induced mechanism. Ivanisenko [27] proposed that shear deformation drove the transformation from bcc to fcc in Fe-C steel after grains are refined into nano scale. Molecular dynamic simulation confirmed that the formation of nanocrystalline structure and dissolved carbides increased Gibbs free energy, providing driving force from bcc to fcc. Laptaoie [28] stated that the transformation from bcc to fcc was controlled by the stress. The fcc structure is not stable because it has a high energy make the transformation from bcc to fcc is reversible. It will transforme back to bcc in the condition of room temperature and unloading. Phase transformation is under formation induced mechanism according to above discussion.

A narrow transition zone of 3-5 $\mathrm{nm}$ happened in the interface of the WEA and matrix showed in Ref. [4] explained that the ordered crystals transformed into a less ordered and then a disordered amorphous phase. An average size of nanocrystallites observed in the amorphous is $5 \mathrm{~nm}$ implies that may exist a transformation size. But this critical size in microstructural changes cannot be captured during RCF. When the size of nanocrystallite reached a critical value, it would preferentially become amorphous. Partial amorphization lead to the amorphous phase and nanocrystallites coexist in the WEA, as shown in Fig. 3(b). A full amorphization occurs when the energy of the system is sufficient for all nanocrystallites to reach a critical size.

\section{Conclusion}

The microstructural changes under RCF is a complex process. We can draw the following conclusions based on the above analysis:

1) The hardness of WEA increased significantly due to the refinement of grains.

2) WEA involves not only nanocrystallization, but amorphization and phase transformation as well. It consists of an amorphous phase with nano martensite, austenite and carbides embedded interior. The content of austenite in WEA is $20 \%$ and in the matrix is $2 \%-3 \%$. The content of austenite in the WEA was increased 10 times compared to the matrix.

3) The nanocrystallization was initiated under large shear plastic deformation. When the grain size were refined into 3-5 $\mathrm{nm}$, the amorphization occurred. The amorphization is partial, leading to the coexistence of nanocrystallites and an amorphous phase. During this process, a phase transformation from martensite to austenite were stimulated under the large deformation.

\section{References}

[1] J.H. Kang, B. Hosseinkhani, P.E.J. Rivera-Diazdel-Castillo, Mater. Sci. Technol. 28, 44-49 (2012)

[2] W. Holweger, M. Wolf, D. Merk, et al, Tribol. Trans. 58, 59-69 (2015)

[3] S.X. Li, Y.S. Su, X.D. Shu, J.J. Chen, Wear 380381, 146-153 (2017)

[4] Y.S. Su, S.X. Li, S.Y. Lu, et al, Int. J. Fatigue 105, 160-168 (2017)

[5] M.H. Evans, Mater. Sci. Technol. 11, 1-37 (2016)

[6] J. Lai, K. Stadler, Wear 364-365, 244-256 (2016)

[7] M.H. Evans, A.D. Richardson, L. Wang, et al, Tribol. Int. 75, 87-97 (2014)

[8] L.B. Wan, S.X. Li, S.Y. Lu, et al, Wear 396-397, 126-134 (2018)

[9] M.H. Evans, J.C. Walker, C. Ma, et al, Mater. Sci. Eng. A 570, 127-134 (2013)

[10] H. Harada, T. Mikami, M. Shibata, et al, ISIJ. Int. 45, 1897-1902 (2005)

[11] G. Baumann, K. Knothe, H.J. Fecht, Nanostruct. Mater. 9, 751-754 (1997)

[12] W. Solano-Alvarez, E.J. Pickering, M.J. Peet, et al, Acta Mater. 121, 215-226 (2016)

[13] B. Gould, A. Greco, Tribol Lett. 62, 1-14 (2016)

[14] A. Warhadpande, F. Sadeghi, R.D. Evans, Tribol. Trans. 56, 349-358 (2013)

[15] J. Lai, K. Stadler, Wear s364-365, 244-256 (2016)

[16] Š. Viktorija, S. Alexander, L. Wang, et al, Int. J. Fatigue 100, 148-158 (2017)

[17] J.H. Kang, B. Hosseinkhani, C.A. Williams, et al, Scripta Mater. 69, 630-633 (2013)

[18] J.H. Kang, R.H. Vegter, Mater. Sci. Eng. A 607, 
328-333 (2014)

[19] Q. Wei, D. Jia, E. Ma, K.T. Ramesh, Appl. Phys. Lett. 81, 1240-1242 (2012)

[20] L.M. Dougherty, E.K. Cerreta, E.A. Pfeif, et al, Acta. Mater. 55, 6356-6364 (2007)

[21] Y. Kadin, M.Y. Sherif, Int. J. Fatigue 96, 114-126 (2017)

[22] A. Grabulov, U. Ziese, H.W. Zandbergen, Scripta Mater. 57, 635-638 (2007)

[23] Y. Zhou, J.F. Peng, Z.P. Luo, et al, Wear 362-363, 8-17 (2016)

[24] M. Steenbergen, R. Dollevoet, 47, 361-372 (2013)

[25] G. Baumann, H.J. Fecht, S. Liebelt, Wear 191, 133140 (1996)

[26] A. Pyzalla, L. Wang, E. Wild, T. Wroblewski, Wear 251, 901-907 (2001)

[27] Y. Ivanisenko, I. MacLaren, X. Sauvage, et al, Acta. Mater. 554, 1659-1669 (2006)

[28] A. Latapie, D. Farkas, Model. Simul. Mater. Sci. Eng. 11, 745-753 (2003) 